\title{
USO DE FÁRMACOS NA SÍNDROME DE HIPERATIVIDADE COM DÉFICIT DE ATENÇÃO
}

\author{
ANA GUARDIOLA *, ALEX RESENDE TERRA**, \\ KAISSARA RICORDI PEREIRA ***, NEWRA TEUECHEA ROTTA ****
}

\begin{abstract}
RESUMO - A sindrome de hiperatividade com déficit de atenção (SHDA) constitui distúrbio comum na infância, podendo advir de fatores exógenos ou endógenos, os quais são responsáveis por comprometimento cerebral, manifestando-se por alterações funcionais dos sistemas motores, perceptivos, cognitivos e do comportamento, comprometendo a aprendizagem de crianças com potencial intelectual adequado. Os autores estudam a açāo de fármacos estimulantes e antidepressivos na SHDA, usando como parâmetro de eficácia as provas de persistência motora.
\end{abstract}

PALAVRAS-CHAVE: síndrome de hiperatividade com déficit de atenção, terapêutica medicamentosa.

\section{Use of drugs in attention deficit hyperactivity disorder}

ABSTRACT - Attention deficit hyperactivity disorder (ADHD) is a neurological disorder which is common in the childhood and can be caused by exogenous and endogenous factors, that are responsible for cerebral disorder. This disorder presents a functional alteration of the motor, perceptive, cognition systems and conduct disorders compromising the learning of children with an adequate intellectual potential. The authors study the action of stimulants and antidepressive drugs in the ADHD, using as measure of efficacy the motor persistence tests

KEY-WORDS: attention deficit hyperactivity disorders, drug therapy.

A síndrome de hiperatividade com déficit de atenção (SHDA) constitui distúrbio comum na infância, podendo advir de fatores exógenos ou endógenos, os quais são responsáveis por comprometimento cerebral, manifestando-se por alteraçōes funcionais. Quando as alterações forem orgânicas ou lesionais teremos paralisia cerebral, deficiência mental e/ou epilepsia, podendo esta última também ocorrer por comprometimento de tipo funcional. Essas possibilidades podem coexistir em diferentes combinaçōes. Para entender melhor o quadro de SHDA é necessário que se fale da moderna concepção bioquímica desta síndrome, sendo a proposição principal desta concepção a existência de alteração do mecanismo de transmissão do impulso nervoso ao nível da sinapse. Ocorrem anomalias no metabolismo das catecolaminas, as quais atuam como neurotransmissores. Muitos estudos mostraram a ação terapêutica das anfetaminas e análogos em crianças com SHDA, o que favorece a teoria de que o tratamento medicamentoso se faça principalmente a custa dos estimulantes do sistema nervoso central. $O$ uso de anfetamínicos em crianças $e$ adolescentes não está isento de riscos, por isso tem-se partido para o emprego de outros fármacos como a cafeína, os antidepressivos, pemoline e deanol $3.5,-8$. . A nortriptilina (NTP) é um antidepressivo trićclico tendo estrutura similar a imipramina (IMI). Em tese de mestrado observamos que a "Persistência Motora" do Exame

*Livre Docente em Neurologia Infantil, Professora Adjunta de Neurologia da Fundação Faculdade Federal de Ciências Médicas de Porto Alegre (FFFCMPA); **Doutorando da FFFCMPA; ***Médica Neurologista; ****Livre Docente em Neurologia, Professora Adjunta de Neurologia da Faculdade de Medicina da Universidade de Federal do Rio Grande do Sul (UFRGS). Aceite: 22-maio-1997.

Dra. Ana Guardiola - Departamento de Neurologia, Fundação Faculdade Federal de Ciências Médicas de Porto Alegre - Rua Sarmento Leite 245 - 90050-140 Porto Alegre RS - Brasil. 
Neurológico Evolutivo (ENE) constitui o parâmetro mais adequado para avaliar a ação de fármacos estimulantes na SHDA ${ }^{3}$.

O presente trabalho estuda a ação da IMI, NTP e metilfenidato (MTF) usando como parâmetro da eficácia destes fármacos as provas de persistência motora.

\section{MÉTODOS}

Constituíram-se 4 grupos de crianças: o grupo que fez uso da IMI ( $n=20)$, o do MTF $(n=15)$, o da NTP $(n=15)$ e o placebo $(n=20)$. Foi feito ensaio clínico, cego, randomizado, controlado por placebo.

As crianças eram de ambos os sexos, provinham da rede escolar de ensino e consultaram por SHDA. As crianças foram examinadas no ambulatório de distúrbios de atenção no Instituto de Neurologia da Irmandade Santa Casa de Misericórdia de Porto Alegre, aleatoriamente, por dois examinadores treinados de forma homogênea. Todos os pais e/ou responsáveis concordaram com as condiçōes do experimento, após lhes terem sido esclarecidos os objetivos e os possíveis efeitos colaterais das drogas. Também lhes foi dito que poderiam retirar seus filhos do estudo no momento que desejassem.

Foi usado: IMI, $1 \mathrm{mg} / \mathrm{kg} / \mathrm{dia}$ em uma tomada; MTF, de $0,25 \mathrm{mg} / \mathrm{kg} / \mathrm{dia}$ em duas tomadas; NTP, $1 \mathrm{mg} / \mathrm{kg} /$ dia em uma tomada; e placebo. Todos tinham forma c cor semelhante.

Todas as crianças foram avaliadas através de exame neurológico, $\mathrm{ENE}^{4}$ e eletrencefalograma (EEG). As provas de persistência motora do ENE (1. manter os olhos fechados durante 20 segundos, 2 . manter a boca aberta durante 40 segundos, 3 . manter a lingua protusa, com os olhos abertos, durante 40 segundos, 4 . igual à prova $n^{\circ}$ 3 , com os olhos fechados, 5 . olhar o extremo lateral para a direita, durante 30 segundos, 6 . olhar o extremo lateral para a esquerda, durante 30 segundos, 7. membros superiores estendidos horizontalmente para a frente, dedos afastados, polegares separados por um cm, manter a posição por 30 segundos, olhos fechados.) foi o instrumento usado para avaliar a efićácia dos fármacos utilizados.

Todas as crianças foram submetidas a duas avaliaçōes com intervalo de 30 dias, sendo avaliadas com os mesmos instrumentos.

$\mathrm{Na}$ análise estatística foram usados os testes ANOVA de Friedman e t de Student.

\section{RESULTADOS}

Na Tabela 1 apresentam-se as características demográficas dos grupos em estudo.

Todas as crianças apresentaram exame neurológico normal, exceto no ítem tono muscular, observando-se hipotonia em 5 crianças do Grupo IMI, 4 do Grupo NTP, 4 do grupo MTI e 4 do Grupo Placebo

As Tabelas 2, 3 e 4 mostram os resultados na persistência motora dos grupos IMI, NTP, MTF e placebo.

Em relação ao EEG, nenhum apresentou elementos paroxísticos; no Grupo IMI, 9 crianças apresentaram traçado lento; nos Grupos NTP e MTF, havia 5 EEG lentos em cada grupo e no Grupo Placebo 7 crianças mostravam EEG lento.

Tabela 1. Caracteristicas demográficas das crianças tratados com IMI, MTF, NTP e placebo.

\begin{tabular}{ccccc}
\hline Características & IMI $(\mathrm{n}=20)$ & MTF $(\mathrm{n}=15)$ & NTP $(\mathrm{n}=15)$ & Placebo $(\mathrm{n}=20)$ \\
\hline Sexo masc., $\mathrm{n}^{\circ}(\%)$ & $12(60)$ & $8(53,3)$ & $9(60)$ & $13(65)$ \\
Idade (meses) & 88 & 89 & 90 & 88 \\
Estatura (cm) & 116,30 & 117,34 & 118,20 & 117,34 \\
Peso (Kg) & 21,90 & 21,82 & 21,37 & 21,64 \\
\hline
\end{tabular}


Tabela 2. Estudo comparativo da persistência motora: imipramina x placebo

\begin{tabular}{ccccc}
\hline Grupo & \multicolumn{2}{c}{ Média } & $\mathrm{X} 2$ & $\mathrm{P}$ \\
& antes & depois & & \\
\hline Imipramina & 5 & 7 & 17,19 & $<0,001^{*}$ \\
Placebo & 5 & 5 & 1,80 & \\
\hline
\end{tabular}

Tesie ANOVA de Friedman; * $P$ com significaçăo estatistica.

Tabela 3. Estudo comparativo da persistência motora: nortriptilina x placebo

\begin{tabular}{ccccc}
\hline Grupo & \multicolumn{2}{c}{ Média } & t & P \\
& antes & depois & & $<0,001^{*}$ \\
\hline $\begin{array}{c}\text { Nortriptilina } \\
\text { Placebo }\end{array}$ & 5 & 6,9 & 4,4 & \\
\hline
\end{tabular}

Teste $\mathrm{l}$ de Student; $* P$ com significaçāo estatística

Tabela 4. Estudo comparativo da persistência motora: metilfenidato depois $x$ placebo depois

\begin{tabular}{ccccc}
\hline Grupo & \multicolumn{2}{c}{ Média } & $t$ & P \\
& antes & depois & & \\
\hline $\begin{array}{c}\text { Metilfenidato } \\
\text { Placebo }\end{array}$ & 5 & 7 & 4,5 & $<0,001^{*}$ \\
\hline
\end{tabular}

Teste $t$ de Student; $* P$ com significação estatística

\section{DISCUSSÃO}

A peristência motora constitui importante auxılio no diagnóstico da criança com distúrbio de atençāo e serve como parâmetro para avaliar a ação de fármacos no SHDA ${ }^{3}$.

Com a pesquisa da persistência motora do ENE se obtém o padrão neurológico da capacidade de manter uma postura apropriada, de controlar os impulsos e sustentar uma atençāo adequada.

Muitos autores ${ }^{1-3.5-8}$ estudaram o uso de fármacos em crianças com distúrbio de atenção, salientando a eficácia. Winsberg e col. ${ }^{8}$ compararam a açāo terapêutica da IMl, placebo e dextroanfetamina no tratamento de crianças hiperativas e com comportamento agressivo: $69 \%$ responderam à IMI e $44 \%$ responderam à dextroanfetamina. Conclufram que a IMI parece ser agente eficaz para o controle da hiperatividade e agressividade e constitui droga valiosa para o tratamento da SHDA em crianças.

Quinn e Rapport ${ }^{5}$, em estudo comparativo, prospectivo, de um ano, em 76 meninos hiperativos, usando MTF, IMI e placebo, relataram que os grupos tratados mostraram melhora na sala de aula, diferindo os resultados a curto prazo em relação ao fármaco usado. A IMI foi superior ao placebo, mas menos eficaz que o MTF, particularmente para a medida dos testes cognitivos. Quando usada a longo prazo, a IMI mostrou resposta clínica similar ao MTF, concluindo que a IMI pode ser uma alternativa dos anfetamínicos.

Wilens e col. ${ }^{7}$, estudando a ação da NTP em 37 crianças e 21 adolescentes com SHDA, verificaram açăo benéfica deste fármaco e conclứram que é mais um recurso farmacológico no tratamento de crianças e adolescentes com hiperatividade e déficit de atenção. 
Neste estudo a imipramina, a nortriptilina e o metilfenidato mostraram ser eficazes no tratamento de crianças com SHDA.

\section{REFERENCIAS}

1. Biederman J, Gastfriend D, Jellinek M. Desipramine in the treatment of children with attention deficit disorder. J Clin Psychopharmacol, 1986;6:359-363.

2. Bradley C. The behavior of children receiving benzedrine. Am J Psychiatry 1937;94:577-585.

3. Guardiola A. Uso da imipramina no estudo da hiperatividade com déficit de atenção. Porto Alegre. Tese, Fundaçăo Faculdade Federal de Ciências Médicas de Porto Alegre, 1990.

4. Lefevre AB. Exame neurológico evolutivo do pré-escolar normal. Săo Paulo: Sarvier, 1972.

5. Quinn PO, Rapoport JL. One-year follow-up of hyperactive boys treated with imipramine or methylphenidate. Am J Psychiatry 1975;132:241-245.

6. Stevenson RD, Wolraich ML. Terapia medicamentosa estimulante para crianças com distúrbio de hiperatividade com déficit de atençăo. Clin Pediatr Am Norte 1989;5:1249-1263.

7. Wilens TE, Biederman J, Geist DE, Steingard R. Spencer T. Nortriptyline in the treatment of ADHD: a chart review of 58 cases. J Am Acad Child Adolesc Psychiatry 1993;32:343-349.

8. Winsberg BG, Bialer I, Kupietz S, Tobias J. Effects of imipramine and dextroamphetamine on behavior of neuropsychiatrically impaired children. Am J Psychiatry 1972;128:109-115. 